FORMATION Formation emploi

Revue française de sciences sociales

112 | octobre-décembre 2010

Pêle-mêle

\title{
Les inégalités de parcours scolaires des enfants d'origine maghrébine résultent-elles de discriminations ?
}

Are the unequal school careers of children of North African descent the result of discrimination?

Ist Diskriminierung die Ursache für ungleiche schulische Laufbahnen von

Kindern mit Maghreb-Immigrationshintergrund?

Las desigualdades de itinerarios escolares de los niños de origen magrebí ¿son el resultado de discriminaciones?

\section{Alain Frickey}

\section{OpenEdition}

Journals

Édition électronique

URL : http://journals.openedition.org/formationemploi/3156

ISSN : 2107-0946

\section{Éditeur}

La Documentation française

Édition imprimée

Date de publication : 30 décembre 2010

Pagination : 21-37

ISSN : 0759-6340

\section{Référence électronique}

Alain Frickey, «Les inégalités de parcours scolaires des enfants d'origine maghrébine résultent-elles de discriminations ? », Formation emploi [En ligne], 112 | octobre-décembre 2010, mis en ligne le 10 janvier 2013, consulté le 30 octobre 2020. URL : http://journals.openedition.org/formationemploi/ 3156

(c) Tous droits réservés 


\title{
Les inégalités de parcours scolaires des enfants d'origine maghrébine résultent-elles de discriminations?
}

Alain Frickey*

\begin{abstract}
L'appartenance à l'un ou l'autre sexe atténue ou renforce les inégalités de parcours scolaires des jeunes originaires d'Europe du Sud et du Maghreb : d'une manière générale, les jeunes femmes issues de l'immigration réussissent mieux à l'école que leurs homologues de sexe masculin.
\end{abstract}

S'il n'y a de sociologie que des rapports inégaux et des figures de la différence, la question est bien pour le sociologue de mettre en avant un principe explicatif, c'est-à-dire de faire des choix entre ce qu'il considère être « la contradiction principale » et toutes les « contradictions secondaires ». Ces choix, érigés en système de différences, masquent d'autres différences ignorées ou, au mieux, soumises au principe explicatif dominant (Passeron, 1991). Il n'est pas d'exemple plus frappant de cette soumission au principe explicatif dominant que celui de la sociologie de l'éducation qui s'est constituée, dans les années 60, autour de la question des inégalités sociales devant l'école. Elle mesurait les inégalités de réussite ou d'accès aux différents niveaux d'enseignement à l'aune quasi exclusive de l'origine sociale.

Dans ce contexte, les inégalités de sexe à l'école étaient considérées comme secondaires; elles faisaient de la figuration, selon l'expression de C. Marry (2001). Soumises aux différenciations de classes, elles n'étaient évoquées qu'à titre accessoire car c'étaient bien les différences de classe qui «commandaient l'ordre de l'analyse », alors que le sexe ne faisait que l'affiner dans le cadre de chaque milieu social. Bref, le sexe n'était qu'une "différence dans la différence » (Passeron, Singly, 1984). Assez paradoxalement, ce n'est que vers la fin des années 80 que les inégalités de sexe ont commencé à susciter un intérêt croissant ; alors même que, dès le début des années 70 , les filles avaient rattrapé leur retard scolaire sur les garçons et les avaient même dépassés pour ce qui est du nombre de bacheliers, référence centrale du système scolaire français. Les progrès scolaires féminins sont apparus au grand jour, même si ces avancées féminines ont toujours été réalisées dans l'ordre des inégalités

* Alain Frickey est sociologue à l'URMIS (Unité de recherche Migration et Sociétél), chargé de recherches CNRS. Ses travaux portent sur la transition de l'école à l'emploi des jeunes issus de l'immigration, les discriminations raciales et les différences de sexe. 
sociales et si des inégalités d'orientation ont persisté entre les sexes. Toujours est-il qu'il était devenu difficile d'ignorer les effets du sexe, au centre de nombre d'analyses, au même titre que l'origine sociale (DuruBellat, 1990 ; Baudelot et Establet, 1991).

Au milieu des années 90, de nouvelles inégalités liées à l'origine nationale ou à la condition d'enfant d'immigré sont apparues dans le champ sociologique. Elles ont été longtemps ignorées. D'abord parce que considérées comme faisant partie intégrante des inégalités sociales : il n'y avait pas lieu de distinguer les cursus scolaires des jeunes issus de l'immigration, parce que ces derniers, dans leur immense majorité, étaient tout à fait emblématiques de la classe ouvrière. Les inégalités qui les frappaient étaient d'abord celles qui affectaient les couches populaires dans leur ensemble. Elles faisaient d'autant moins l'objet d'investigations que l'appareil statistique français permettait difficilement d'identifier les jeunes d'origine étrangère ; le manque de données s'expliquant également par une absence de légitimation de l'objet d'études par la communauté scientifique. Malgré un certain nombre de travaux précurseurs (Zirotti, 1980 ; Bastide, 1984 ; Zeroulou, 1988), il faut attendre le milieu des années 90 pour que les problématiques centrées sur les immigrés se constituent en un domaine de recherche. C'est à cette époque que Vallet et Caille introduisent la nationalité de l'élève et son lieu de naissance comme critères pertinents dans l'étude des scolarités en France. (Vallet et Caille, 1996). Dans l'impossibilité de séparer les élèves immigrés des élèves enfants d'immigrés, ils ont observé la population des élèves étrangers ou issus de l'immigration. Leur travail témoigne d'écarts importants dans la comparaison brute des performances et des parcours scolaires des enfants d'immigrés et ceux des enfants d'origine française, notamment s'agissant du critère du redoublement au cours de la scolarité primaire.

Les inégalités scolaires observées par Vallet et Caille relèvent essentiellement, selon eux, d'un effet de structure; en d'autres termes, elles sont dues aux caractéristiques associées aux populations immigrées qui ont, plus fréquemment que les jeunes d'origine française, des parents ouvriers, peu diplômés, et sont issus de familles nombreuses. Ce sont ces caractéristiques qui sont corrélées à un risque accru de difficultés scolaires et non le fait d'être d'origine étrangère ${ }^{1}$. Dans ce modèle, les différences de sexe interviennent comme variable explicative, au même titre que l'origine. Les auteurs soulignent que les filles, toutes choses égales par ailleurs, connaissent à l'école primaire de meilleures scolarités que les garçons, mais les deux dimensions sexe et origine nationale ne sont jamais croisées.

L'objet de cet article est d'étudier les parcours scolaires des jeunes issus de l'immigration. Pour ce faire, il s'appuie sur les principaux points de bifurcation et d'orientation générés par le système scolaire, en tenant compte des dimensions du sexe et de l'origine nationale. Si des inégalités sont mises au jour, se cumulent-elles pour les jeunes hommes issus de l'immigration? Les destinées scolaires des jeunes femmes d'origine immigrée sont-elles davantage structurées par leur sexe que par leur origine? L'origine immigrée, sans autre précision relative au pays d'origine, peut-elle encore constituer une catégorie d'analyse pertinente? Les inégalités de parcours peuvent-elles être assimilées à des discriminations ? D'autre part, quel est le point de vue des jeunes sur les orientations qu'ils ont la plupart du temps subies?

Pour répondre à ces questions, nous comparerons les parcours scolaires des jeunes dont les parents ont émigré en France à ceux des descendants de natifs, sous le double point de vue de l'origine nationale et du sexe. Toutefois, nous limiterons notre comparaison aux jeunes issus de la population ouvrière ${ }^{2}$. Il s'agit

\footnotetext{
${ }^{1}$ Ces résultats ont fait l'objet de nombreux débats, notamment sur la signification des performances égales ou supérieures des jeunes issus de l'immigration, toutes choses égales par ailleurs. Ce type de raisonnement implique que les jeunes des familles immigrées sont marqués par les caractéristiques sociales qui leur sont associées et qu'une fois que ces conditions sont neutralisées ou égalisées, leurs performances à l'école ne diffèrent pas de celles des jeunes issus de familles non immigrées.

${ }^{2}$ Il semblerait cependant que, du point de vue des parcours scolaires, la prise en considération de l'origine ouvrière n'ait pas les mêmes effets dans la population d'origine française et dans celle originaire d'Afrique du Nord. Les jeunes d'origine française nés de parents ouvriers présentent une structure de sortie du système scolaire plutôt en retrait par rapport à celle constatée sur l'ensemble de la cohorte: plus fréquemment non diplômés et, en proportion, moins nombreux à détenir un diplôme de l'enseignement supérieur. Paradoxalement, pour les jeunes d'origine maghrébine, la prise en compte de la seule catégorie ouvrière a un effet inverse : les enfants d'ouvriers présentent une structure de sortie plus favorable par rapport à celle affichée par l'ensemble de la cohorte issue de l'immigration (cf. tableau 1). On sait que la jeunesse issue de l'immigration maghrébine est une jeunesse populaire, où les emplois d'ouvriers sont largement majoritaires, mais on peut faire l'hypothèse que l'appartenance à la catégorie
} 


\section{Encadré 1}

\section{Du panel « Génération 98 » aux entretiens biographiques}

Cet article s'appuie sur l'enquête "Génération 98 » du Cereq, qui a interrogé, en 2001, un échantillon de 54000 jeunes de tous niveaux sortis de la formation initiale trois ans plus tôt. Cette enquête avait pour objectif principal d'analyser les premières années de la vie active en fonction du niveau et de la spécialité de formation atteints; elle comportait également des informations sur le déroulement de la scolarité secondaire. D'autre part, cette enquête permettait de différencier ces jeunes selon l'origine nationale de leurs parents. L'article s'appuie également sur des données plus qualitatives rassemblées à partir d'entretiens semi-directifs auprès de 65 jeunes d'origine nord africaine et issus du panel "Génération 98 ». Ces jeunes ont été sélectionnés après avoir répondu, dans l'enquête G98, s'être senti discriminés dans l'emploi en raison de leur origine. Nous nous sommes par ailleurs efforcés de couvrir l'ensemble des trajectoires d'insertion rencontrées par ces jeunes (de l'insertion rapide et durable à la persistance de la précarité). Au cours des entretiens, ces jeunes ont été invités à revenir sur leur trajectoire professionnelle, familiale, résidentielle mais aussi sur leur parcours scolaire, leur orientation, l'existence ou non de discriminations à l'école, le contexte familial, etc. ( ${ }^{*}$.

Nous distinguons les jeunes dont les deux parents sont nés dans un pays du Maghreb (Algérie, Maroc, Tunisie) ou en Europe du Sud (principalement Portugal, mais aussi Espagne et Italie), sans avoir la nationalité française, de leurs homologues dont les deux parents sont nés en France ou nés français dans un pays étranger $\left({ }^{* *}\right)$.

Enfin, la construction de la catégorie des descendants d'immigrés ne va pas de soi. Elle agrège en effet des jeunes qui sont nés en France et d'autres, nés à l'étranger et arrivés en France à un âge plus ou moins avancé, confrontés à une nouvelle langue, à un nouveau système scolaire, à de nouvelles méthodes pédagogiques... Dans le cas de la population originaire d'Afrique du Nord, les jeunes nés à l'étranger sont particulièrement nombreux et totalisent $28 \%$ de l'ensemble de la population. Dans le cadre de l'étude des scolarités des jeunes originaires d'Afrique du Nord, il faut donc veiller à séparer ceux nés en France et ceux nés à l'étranger, faute de quoi certains indicateurs, comme par exemple le taux de redoublement au cours du primaire, peuvent être biaisés. Cette précaution méthodologique n'est pas indispensable pour les jeunes issus de l'immigration sud européenne car seulement $5 \%$ d'entre eux sont nés hors de France.

$\left.{ }^{*}\right)$ : Projet ANR «Discriminations ressenties et inégalités sociales : des données statistiques aux récits biographiques ». Coordination A. Frickey, juin 2010.

$\left.{ }^{* *}\right)$ : Une nationalité étrangère n'est pas directement définie dans l'enquête. Seul est renseigné le pays de naissance de chacun des deux parents, complété par un critère de nationalité dans le cas où la personne est née à l'étranger. Nous limitons l'analyse aux jeunes issus de l'immigration maghrébine et sud européenne pour des raisons d'effectifs.

ainsi de contrôler les effets de structure potentiellement importants dus à la forte proportion de familles ouvrières au sein des populations d'origine immigrée? Nous reconstituerons leurs parcours scolaires de

ouvrière n'a pas le même sens dans la population immigrée nord africaine, dans la mesure où l'appartenance au monde ouvrier est récente et coïncide fréquemment avec l'immigration et peut être vécue comme un changement de condition sociale, dynamique que les familles tenteraient de poursuivre en investissant dans les études de leurs enfants.

${ }^{3}$ Lorsque les deux parents sont d'origine nord africaine, $60 \%$ des pères sont ouvriers, contre $21 \%$ pour les jeunes d'origine française. manière chronologique, de l'école primaire, abordée sous l'angle de la fréquence des redoublements, à l'enseignement supérieur; nous nous focaliserons sur les principaux points de bifurcations du système et sur les conséquences pour les enfants d'immigrés en termes de poursuite d'études et d'obtention du diplôme terminal. Ces parcours seront ensuite synthétisés dans une analyse toutes choses égales par ailleurs. Mais au préalable nous présentons une photographie d'ensemble du niveau scolaire des hommes et femmes de la Génération 98, qu'ils (elles) soient d'origine immi- 
grée ou non, telle qu'elle s'est présentée sur le marché du travail.

\section{SELON L'ORIGINE ET LE SEXE, DES NIVEAUX INÉGAUX DE SORTIE DU SYSTĖME ÉDUCATIF}

Très schématiquement, si l'on s'intéresse à la structure des sorties (tableau 1) des enfants d'ouvriers, on observe une surreprésentation des jeunes de l'immigration nord africaine parmi les non-diplômés et une présence moindre parmi les diplômés de l'enseignement supérieur. Ce constat n'est cependant pas valable pour les jeunes originaires des pays de l'Europe du Sud. Ces derniers, aux deux extrêmes de l'échelle scolaire, présentent des taux très comparables à ceux obtenus par la population dite « d'origine française ».

La surreprésentation des jeunes d'origine maghrébine parmi les non-diplômés se vérifie pour ceux d'entre eux nés en France ainsi que pour ceux nés à l'étranger. Cependant, comme on pouvait l'anticiper, elle est particulièrement marquée pour ces derniers ${ }^{4}$. L'intérêt de la prise en compte du pays de naissance ne s'arrête pas à une diminution de l'échec scolaire corrélée à l'ancienneté de l'émigration. Elle permet également de mettre au jour des différences de comportements des jeunes filles maghrébines et de leurs familles visà-vis de l'institution scolaire. L'arrivée en France de ces jeunes filles, en cours de scolarité, est synonyme d'une absence de certification supérieure à celle de leurs homologues masculins (24\% contre 19\%). Socialisées en France, la sortie sans diplôme de ces jeunes filles diminue drastiquement. Avec un taux de non-certification de $11 \%$, elles surclassent leurs homologues masculins et se conforment au modèle scolaire dominant en France.

En revanche, les jeunes hommes nés en France de parents maghrébins restent très vulnérables face à l'échec scolaire : distancés sur ce point par les filles, leurs positions ne s'améliorent pas par rapport à leurs

\footnotetext{
${ }^{4} 21 \%$ des jeunes nés au Maghreb sortent du système scolaire sans aucun diplôme, contre $16 \%$ pour les jeunes nés en France de parents immigrés d'Afrique du nord, chiffre deux fois plus élevé que celui enregistré pour les jeunes d'origine sud européenne.
}

homologues nés à l'étranger et, pour la plupart, arrivés en cours de scolarité. Si l'on élargit la notion d'échec, en considérant l'ensemble des jeunes qui ont quitté le système scolaire sans achever le cycle d'études secondaires, les résultats décrits ci-dessus demeurent identiques. On enregistre une diminution de moitié de la non-certification pour les femmes d'origine maghrébine nées en France comparées à celles arrivées après leur naissance (14\% contre $28 \%$ ). À l'opposé, les hommes sont proportionnellement aussi nombreux à

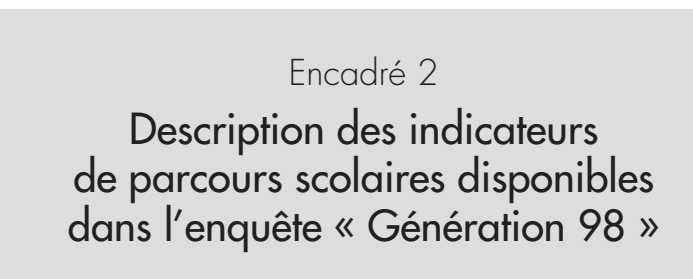

L'enquête "Génération 98 » permet d'évaluer les parcours au sein de l'enseignement secondaire en fonction de l'origine nationale et du sexe des jeunes interrogés. Les indicateurs utilisés sont l'âge de la première inscription en $6^{e}$, la classe de $3^{e}$ suivie lgénérale, technologique ou autre...), la classe de seconde suivie et le type ou la série du baccalauréat présenté. Les jeunes les plus qualifiés ont arrêté leurs études vers 25 ans et ont donc été scolarisés en moyenne sept ou huit ans avant ceux sortant du système éducatif sans aucun diplôme vers l'âge de 18 ans ou avant. Puisque la "Génération 98 » rassemble plusieurs tranches d'âge, ces indicateurs devraient, en toute rigueur, être systématiquement croisés avec l'âge de sortie ou, à défaut, avec le niveau de sortie; pour autant que l'âge moyen, à chaque niveau de sortie, soit relativement homogène pour les différentes populations comparées. Cette homogénéité est dans l'ensemble plutôt forte, pour chaque niveau de sortie; les âges moyens se différenciant assez peu pour chaque sexe en fonction de l'origine nationale des parents. II est vrai que les jeunes d'origine maghrébine sont, dans l'ensemble, un peu plus âgés à chaque niveau de sortie, ce qui traduit des parcours scolaires davantage marqués par les redoublements (cf. infra). 
Tableau 1

Niveau atteint par les hommes et les femmes selon l'origine nationale des parents

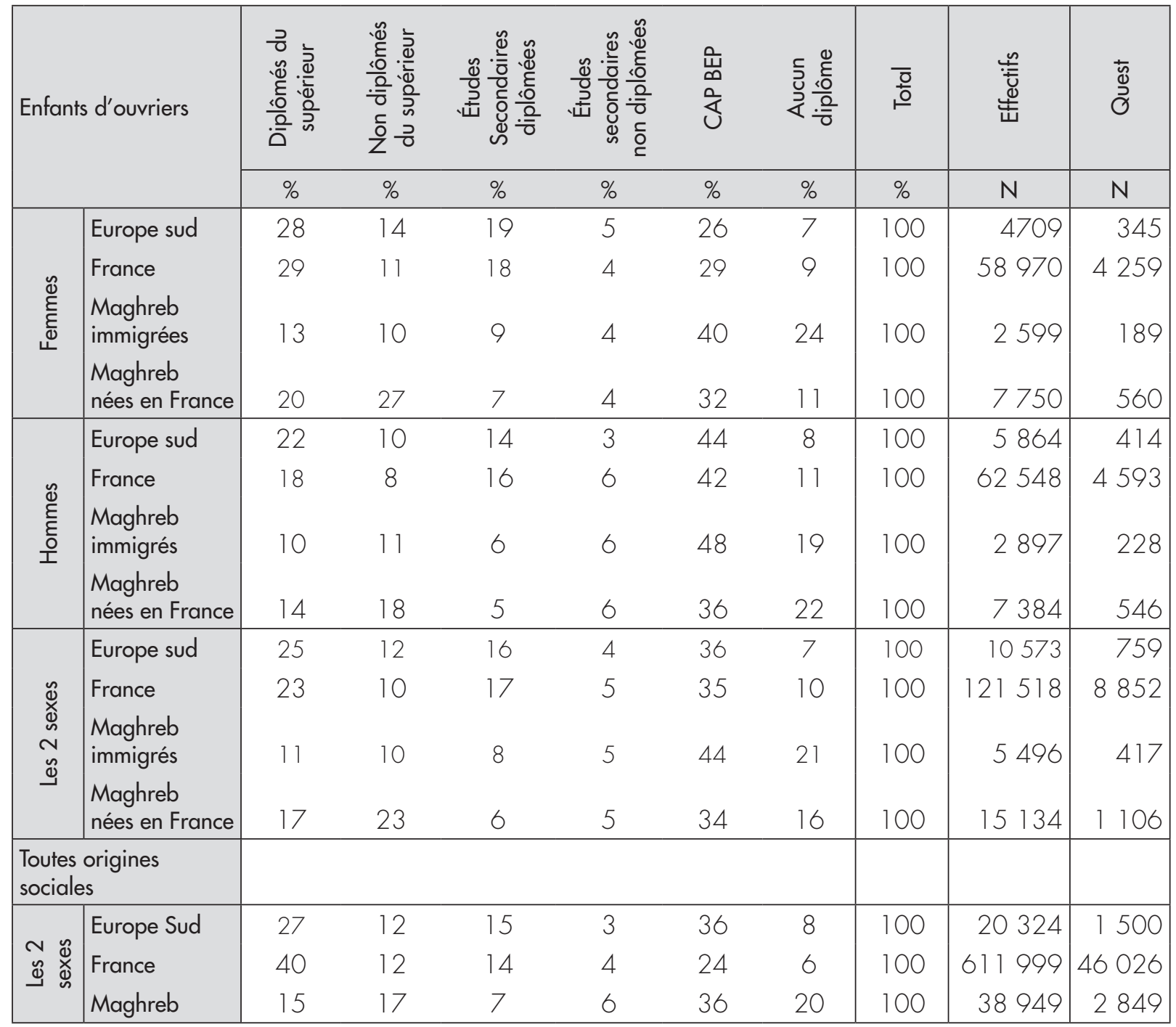

Lecture : $28 \%$ des jeunes femmes originaires d'Europe du Sud ont quitté le système éducatif avec un diplôme de l'enseignement supérieur, $14 \%$ après avoir fréquenté un établissement du supérieur mais sans obtenir de diplôme, etc.

Sigles : CAP : Certificat d'aptitude professionnelle. BEP : Brevet d'études professionnelles.

Source : Exploitation originale Enquête « Génération 98 » du Céreq.

ne pas terminer les études entreprises, qu'ils soient nés en France (28\%) ou à l'étranger (25\%).

À l'autre extrémité de l'échelle des diplômes, les jeunes, nés de parents ouvriers, d'origine française ou sud européenne, sont plus fréquemment diplômés de l'enseignement supérieur ( $25 \%$ d'entre eux sont dans ce cas) que les jeunes de parents nord africains, qu'ils soient nés en France (17\%) ou à l'étranger (11\%).
De manière constante, les jeunes femmes sont proportionnellement plus nombreuses à être diplômées que leurs collègues masculins. Ce premier indicateur, très général, laisse entrevoir qu'à l'école, quelle que soit l'origine nationale, les différences de «performances » entre garçons et filles persistent ; elles sont même renforcées par une origine nord africaine, dès lors que la socialisation scolaire s'est déroulée en France dès le plus jeune âge. 


\section{DES REDOUBLEMENTS FRÉQUENTS AU PRIMAIRE POUR LES JEUNES D'ORIGINE OUVRIÈRE}

En l'absence de toute mesure des inégalités scolaires à l'entrée du primaire, le critère du redoublement synthétise le parcours primaire dans son ensemble. Devenu relativement rare au cours du primaire, le redoublement concerne moins d'un quart de la population. Malgré cet inconvénient, le fait d'avoir redoublé ou non la scolarité primaire apporte des informations intéressantes. On sait notamment que l'âge d'entrée en sixième, et donc la manière dont s'est déroulée la scolarité à l'école primaire, est un des facteurs déterminants de la réussite au collège ${ }^{5}$. Dès la fin du primaire, les jeunes originaires - par leurs parents - des pays du Maghreb ont été fortement affectés par une ou plusieurs mesures de redoublement de classe ${ }^{6}$. Globalement, la moitié des enfants d'ouvriers originaires du Maghreb sont en retard lors de leur première entrée en $6^{\mathrm{e}}$. En faisant abstraction des jeunes arrivés en France en cours de scolarité, la proportion de redoublants à l'école primaire parmi les enfants d'ouvriers d'origine nord africaine diminue nettement. Elle reste cependant plus élevée que celle enregistrée pour les jeunes de même origine sociale et nés de parents français (43\% contre $28 \%$ pour les femmes et $45 \%$ contre $36 \%$ pour les hommes). Certes, dans les trois populations étudiées, les jeunes filles sont systématiquement moins concernées que les garçons par le redoublement. Toutefois, au vu de ce premier indicateur, les parcours des jeunes femmes originaires du Maghreb se rapprochent assez fortement de ceux de leurs homologues masculins.

Le collège unique, au moins en apparence, ne différencie pas les élèves en fonction de leurs origines nationales : à l'entrée en troisième, les variations d'une origine à l'autre sont faibles et, dans tous les

\footnotetext{
$593 \%$ des élèves entrés en $6^{\mathrm{e}}$ à 10 ans et moins parviennent en $4^{\mathrm{e}}$ sans redoubler, contre $63 \%$ des élèves de 12 ans à cette même entrée en $6^{\text {e }}$. (MEN Note d'Information 00.54).

${ }^{6}$ Dans l'enquête « Génération 98 ", le taux de redoublement dans l'enseignement primaire se déduit de l'âge lors de la première inscription en $6^{\mathrm{e}}$, qui est la variable figurant dans le questionnaire. Les jeunes en retard sont considérés comme ayant redoublé leur scolarité primaire.
}

cas de figure ${ }^{7}$, une forte majorité d'élèves intègre une $3^{\mathrm{e}}$ générale. En revanche, les écarts entre hommes et femmes sont plus marqués et toujours à l'avantage de ces dernières. Cette situation préfigure leur prépondérance dans les filières générales du lycée, puis dans l'enseignement supérieur. Mais, à sexe et origine identiques, un retard à l'entrée en $6^{\mathrm{e}}$ est synonyme d'une forte réduction de chances d'accéder à une $3^{\mathrm{e}}$ générale et très probablement d'une orientation vers l'enseignement professionnel.

\section{L'ORIENTATION APRÈS LA $3^{E}$ : UNE BIFURCATION QUI DIFFÉRENCIE PLUS LES SEXES QUE LES ORIGINES}

En cours de $3^{e}$ générale, les familles émettent des vœux relatifs à la poursuite de la scolarité de leurs enfants. Les années 1985 à 1990 ont été marquées par une intensification des vœux des familles en faveur des études longues. Y. Birnbaum et A. Kieffer (2005), exploitant le panel d'élèves de 1995 de la DEP, montrent qu'à milieu social équivalent, les parents en provenance d'Afrique du nord auraient des aspirations plus élevées que celles de leurs homologues d'origine française ou portugaise, en privilégiant les études longues pour leurs enfants. Les parents d'origine portugaise opteraient plutôt pour les filières professionnelles. La mise en relation des aspirations et des parcours effectués révèle l'existence de " décalages, réajustements et ruptures »: les jeunes d'origine portugaise ont ainsi davantage tendance à prolonger leurs études vers le supérieur que ne le souhaitaient leurs parents ; les jeunes issus de familles maghrébines quant à eux, proches des aspirations parentales, présentent des parcours décalés par rapport à ces ambitions; et ce principalement en raison de leur orientation vers des filières technologiques et professionnelles.

De fait, pour les sortants de la "Génération 98 », à l'issue de la classe de $3^{\text {e }}$, la majorité des enfants d'ouvriers d'Afrique du nord a été orientée vers l'ensei-

\footnotetext{
${ }^{7}$ À l'exception des jeunes hommes nés au Maghreb qui ne sont que $45 \%$ à intégrer une $3^{e}$ générale (contre $56 \%$ pour les jeunes filles elles-mêmes nées au Maghreb).
} 
Tableau 2

Classe suivie après la troisième selon l'origine nationale et le sexe

\begin{tabular}{|c|c|c|c|c|c|c|c|c|c|}
\hline & \multirow[t]{2}{*}{ Enfants d'ouvriers } & $\begin{array}{c}\text { Une } \\
\text { seconde } \\
\text { générale }\end{array}$ & $\begin{array}{c}\text { Une } \\
\text { seconde } \\
\text { techno. }\end{array}$ & $\begin{array}{c}\text { Une } \\
\text { première } \\
\text { année de } \\
\text { BEP }\end{array}$ & $\begin{array}{c}\text { Une } \\
\text { première } \\
\text { année de } \\
\text { CAP }\end{array}$ & $\begin{array}{l}\text { Autres } \\
\text { (orientés } \\
\text { avant } 3^{\mathrm{e}} \text { ) }\end{array}$ & Total & Effectifs & Quest. \\
\hline & & $\%$ & $\%$ & $\%$ & $\%$ & $\%$ & $\%$ & $\mathrm{~N}$ & $\mathrm{~N}$ \\
\hline \multirow{4}{*}{ 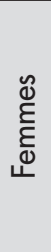 } & Europe Sud & 44 & 7 & 36 & 7 & 6 & 100 & 4709 & 345 \\
\hline & France & 41 & 6 & 37 & 9 & 7 & 100 & 58970 & 4259 \\
\hline & Maghreb immigrées & 22 & 3 & 45 & 10 & 20 & 100 & 2599 & 189 \\
\hline & $\begin{array}{l}\text { Maghreb } \\
\text { nées en France }\end{array}$ & 39 & 5 & 43 & 6 & 7 & 100 & 7750 & 560 \\
\hline \multirow{4}{*}{$\begin{array}{l}\mathscr{\varrho} \\
\sum_{\xi}^{E} \\
\text { 오 }\end{array}$} & Europe Sud & 24 & 11 & 32 & 15 & 18 & 100 & 5864 & 414 \\
\hline & France & 22 & 10 & 38 & 18 & 12 & 100 & 62548 & 4593 \\
\hline & Maghreb immigrés & 13 & 9 & 42 & 18 & 19 & 100 & 2897 & 228 \\
\hline & $\begin{array}{l}\text { Maghreb } \\
\text { nés en France }\end{array}$ & 26 & 7 & 43 & 10 & 14 & 100 & 7384 & 546 \\
\hline
\end{tabular}

Lecture : $44 \%$ des jeunes femmes originaires d'Europe du Sud, sorties du système éducatif en 1998, avaient fréquenté une classe de seconde générale, contre $24 \%$ des hommes de la même origine, etc.

CAP : Certificat d'aptitude professionnelle. BEP : Brevet d'études professionnelles.

Source : Exploitation originale Enquête « Génération 98 » du Cereq.

gnement professionnel. Les secondes générales ou technologiques ne constituent l'orientation majoritaire que pour les jeunes filles issues de l'immigration en provenance d'Europe du Sud. Pour tous les autres jeunes d'origine ouvrière, l'enseignement professionnel (première année de BEP ou de CAP ou orientation vers des filières professionnelles avant la classe de $3^{\mathrm{e}}$ ) est majoritaire. Néanmoins, dans les populations étudiées, les jeunes filles sont toujours nettement plus nombreuses dans l'enseignement général que leurs homologues masculins. Cette tendance se maintient, quel que soit le niveau d'études atteint. D'autre part, la proportion de jeunes ayant fréquenté une seconde générale est d'autant plus forte que le niveau de sortie est élevé. Pour autant, l'orientation des jeunes d'origine nord africaine diffère-t-elle significativement de celle constatée pour les jeunes d'origine française ou sud européenne ? Si le critère de différentiation se focalise sur la proportion de jeunes admis en seconde générale, les écarts avec les descendants de natifs sont minimes pour les jeunes femmes et à l'avantage des hommes d'origine maghrébine, lorsque l'on compare des situations sociales homogènes sinon identiques ${ }^{8}$. Le critère de l'admission dans une seconde générale est important. En effet, l'orientation vers la voie professionnelle est une orientation subie dans la plupart des cas. Les travaux de Caille, fondés sur le panel d'élèves du MEN (ministère de l'Éducation nationale) suivis depuis leur entrée au collège, en 1995, montrent, d'une part, qu'en fin de $3^{\text {e }}$ le sentiment d'orientation contrainte concerne $44 \%$ des lycéens technologiques et $63 \%$ des lycéens professionnels ; d'autre part, que les enfants d'immigrés sont confrontés à un refus de l'orientation choisie dans des proportions plus élevées que les autres élèves. L'orientation vers l'enseignement professionnel procède d'un niveau scolaire insuffisant et reconnu comme tel par les trois quarts des jeunes maghrébins ayant fait l'objet d'une telle orientation, même si les enfants d'immigrés expriment un sentiment d'injustice plus prononcé. À cet égard pourtant, les analyses raisonnant toutes choses égales par ailleurs ne permettent pas de mettre en

\footnotetext{
${ }^{8}$ On sait que, bien souvent, les familles ouvrières maghrébines appartiennent aux franges les plus paupérisées et précarisées du monde ouvrier.
} 
évidence des discriminations envers les jeunes issus de l'immigration en matière d'appréciation du niveau scolaire, que ce soit à l'entrée en $6^{\mathrm{e}}$ ou à l'orientation en fin de $3^{\mathrm{e}}$ (Caille, 2007).

Il n'empêche, ce sentiment d'injustice est une constante. Il est également très fréquemment observé et exprimé dans les entretiens approfondis menés auprès de jeunes d'origine maghrébine ayant répondu à l'enquête Génération 98. Avec un regard rétrospectif et distancié par rapport à leur parcours scolaire ${ }^{9}$, les jeunes d'origine maghrébine orientés vers l'enseignement professionnel gardent un fort sentiment d'injustice, qui n'est que rarement assimilé à une discrimination ${ }^{10}$. Dans le domaine scolaire, l'absence de ressenti discriminatoire n'est pas pour surprendre. Il est plus facile d'évoquer une injustice qu'une discrimination. L'orientation subie est vécue comme une injustice, mais sur le mode individuel; le jeune est seul face à l'institution qui ne l'écoute pas ou qui a d'autres critères de jugement. Il est beaucoup plus difficile d'analyser la décision, même rétrospectivement, en termes de discrimination; celle-ci faisant référence à l'appartenance à un groupe subissant un traitement inégal. D'après les témoignages recueillis, les motifs profonds de ces "orientations forcées» sont parfois vus plutôt comme le résultat d'une appartenance de classe que d'une discrimination liée à l'origine nationale. En d'autres termes, les enseignants, et surtout les services d'orientation, ne prendraient pas vraiment en compte les capacités réelles des élèves. Ils se projetteraient à la place des élèves, en « voulant ou en croyant bien faire ». Ils limiteraient ainsi le champ des possibles des jeunes issus de l'immigration. Ils les orientent en effet vers les filières professionnelles courtes, anticipant ainsi les difficultés matérielles, morales ou financières qui ne manqueraient pas de survenir en cas d'engagement au sein des filières générales longues.

\footnotetext{
${ }^{9}$ Les jeunes interviewés dans le cadre de l'enquête DRIS l'ont été dix ans après leur sortie du système scolaire.

${ }^{10}$ L'hésitation voire la réticence à employer le terme de discrimination est une constante observée tout au long des entretiens biographiques, que les enquêtés se situent dans les champs scolaire, professionnel ou du logement, alors même qu'ils décrivent des situations concrètes de discriminations caractérisées.
}

"Comme j'étais quelqu'un qui était plutôt en retrait, les enseignants se sont dit: "Celui-là, il ne s'en sortira jamais" sans, quelque part, se soucier réellement de ses capacités. Ils ont une image de l'élève, juste en regardant, par exemple, la catégorie socioprofessionnelle des parents (...) Et les enseignants qui ont cette image-là, si vous voulez, pensent bien faire, puisqu'ils se disent: "Il n'aura pas la possibilité de faire d'études parce que ses parents ne sont pas favorisés, donc, quelque part, ce serait bien qu'il aille en lycée professionnel, plutôt vers une voie courte. ” (...). Disons qu'on était jugés... pas vraiment sur nos capacités, mais plus sur une projection que se faisait l'enseignant, en voulant bien faire, quelque part. Mais, si vous voulez, ce n'est pas de son ressort. Moi en tout cas, c'est le discours que je tiens maintenant. » (Mansour, enseignant en comptabilité bureautique, préparant actuellement l'agrégation).

Ce sentiment d'injustice est renforcé par le fait que ces jeunes sont le plus souvent seuls face à l'institution scolaire et aux adultes, en situation d'infériorité. Ils n'ont aucune expérience face au conseiller d'orientation, adulte, qui, en théorie, détient le savoir et qui peut aller jusqu'à exercer une sorte de chantage : « Si tu veux continuer l'école, c'est ça et tu n'as pas le choix. Si tu ne prends pas ça, tu n'arriveras à rien » (propos du conseiller d'orientation rapportés par Kouider, éducateur, orienté en BEP - brevet d'études professionnelles - Productique mécanique). Leurs parents, illettrés le plus souvent, ne sont pas en mesure de s'opposer aux décisions ou propositions d'orientations ; alors que d'autres jeunes, pas nécessairement meilleurs scolairement, parviennent, grâce à l'intervention des familles, à mettre en place des stratégies d'évitement de l'enseignement professionnel, toujours vu comme la voie de garage : " $j$ 'avais peur de tomber dans le professionnel $\gg$ (Sofia, assistante de direction, orientée vers l'enseignement professionnel, et qui a passé ensuite un BTS - brevet de technicien supérieur - Assistante de direction), en opposition à la voie générale, toujours associée à la "normalité » : "après une première d'adaptation, je suis revenue vers le cycle normal » (Saliha, au chômage, orientée vers un BEP Secrétariat). 


\section{L'ORIENTATION APRÈS LA $3^{\text {E }}$ DÉTERMINE LES CHANCES D'OBTENIR UN BACCALAURÉAT}

La voie scolaire empruntée par les jeunes, une fois le collège terminé, va se révéler déterminante pour l'accès au baccalauréat. D'évidence, pour les enfants d'ouvriers, le choix, plus contraint que volontaire, d'un BEP ou d'un CAP à l'issue de la troisième, limite considérablement les chances d'être bachelier; même si les baccalauréats professionnels constituent le prolongement logique de l'enseignement secondaire professionnel. Rétrospectivement, les chances d'être bachelier, après avoir été orienté vers les classes de BEP à la fin de la troisième, sont relativement faibles. Pour l'ensemble des sous-populations considérées, une telle orientation est plutôt synonyme d'une sortie du système scolaire à un niveau inférieur au baccalauréat, avec ou sans diplôme professionnel. De 70 à $81 \%$ des hommes orientés en BEP n'accèdent pas au bac. 53 à $63 \%$ des femmes sont dans le même cas. Ce sont les jeunes descendants de parents nord africains des deux sexes qui accèdent le moins fréquemment au bac professionnel après avoir été orientés en BEP.

L'accès à une seconde générale est très clairement corrélé à l'obtention d'un baccalauréat, pour les femmes comme pour les hommes, qu'ils soient d'origines sud européenne, nord africaine ou française. Les écarts sont ici assez faibles entre les sous-populations étudiées. Notons qu'environ neuf lycéens sur dix de classe de seconde générale de l'enquête « Génération 98 » ont décroché un baccalauréat par la suite.

En revanche, ce qui caractérise les jeunes d'origine nord africaine admis en seconde générale, c'est qu'une forte proportion d'entre eux se réorientent ou sont réorientés vers les filières technologiques, au point que, pour les hommes, les baccalauréats technologiques (41\%) deviennent dominants par rapport aux baccalauréats généraux (39\%). Autrement dit, pour un nombre important de jeunes nés de parents nord africains, le processus d'orientation amorcé à la fin du collège s'est poursuivi au lycée ; cependant, il a pris une forme distincte de celle que connaissent la majorité des élèves qui, à ce stade du cursus secondaire, choisissent généralement entre les différentes séries du baccalauréat général. Ces orientations ou réorientations permanentes confèrent aux scolarités des jeunes d'origine nord africaine un caractère alinéaire, difficilement saisissable dans les interviews précitées ; ici, les chemins de traverses sont en effet multipliés. Ces jeunes n'auraient pas emprunté de leur propre chef ces chemins ; pourtant, ils se voient contraints de les suivre pour rester dans le système scolaire. Le ressentiment à l'égard de l'institution dix ans après la fin des études reste fort. Ce ressentiment est d'autant plus prégnant que l'orientation subie est le plus souvent attribuée à l'obligation de remplir les filières professionnelles ou technologiques. À cela s'ajoute le fait que les services d'orientation sont perçus comme un service qui répartit les élèves en fonction des places disponibles plutôt qu'en vertu de leurs capacités effectives, jugées hâtivement au cours d'entretiens d'orientation trop brefs.

Le choix de la filière du baccalauréat, générale, technologique, professionnelle dépend largement de l'ensemble du processus d'orientation/sélection décrit ici très schématiquement. L'orientation préférentielle des femmes en classe de seconde générale, associée, on vient de le voir, à une forte probabilité d'obtenir un baccalauréat, favorise indéniablement les femmes. Elles sont dès lors et très logiquement plus nombreuses à obtenir un baccalauréat : les écarts sont de l'ordre de 20 points avec les hommes ; et ce indépendamment de l'origine nationale de leurs parents (tableau 3). Cependant, dans ce contexte général, les jeunes nés de parents nord africains se situent en léger retrait par rapport à leurs condisciples d'origine française ou sud européenne.

\section{MUNIS D'UN BACCALAURÉAT, LES JEUNES D'ORIGINE NORD AFRICAINE OPTENT POUR L'ENSEIGNEMENT SUPÉRIEUR...}

Intéressons-nous à présent au devenir des jeunes bacheliers. Tous baccalauréats confondus, les jeunes d'origine maghrébine, hommes comme femmes, sont ceux qui connaissent la plus forte propension à poursuivre des études au sein d'un établissement 


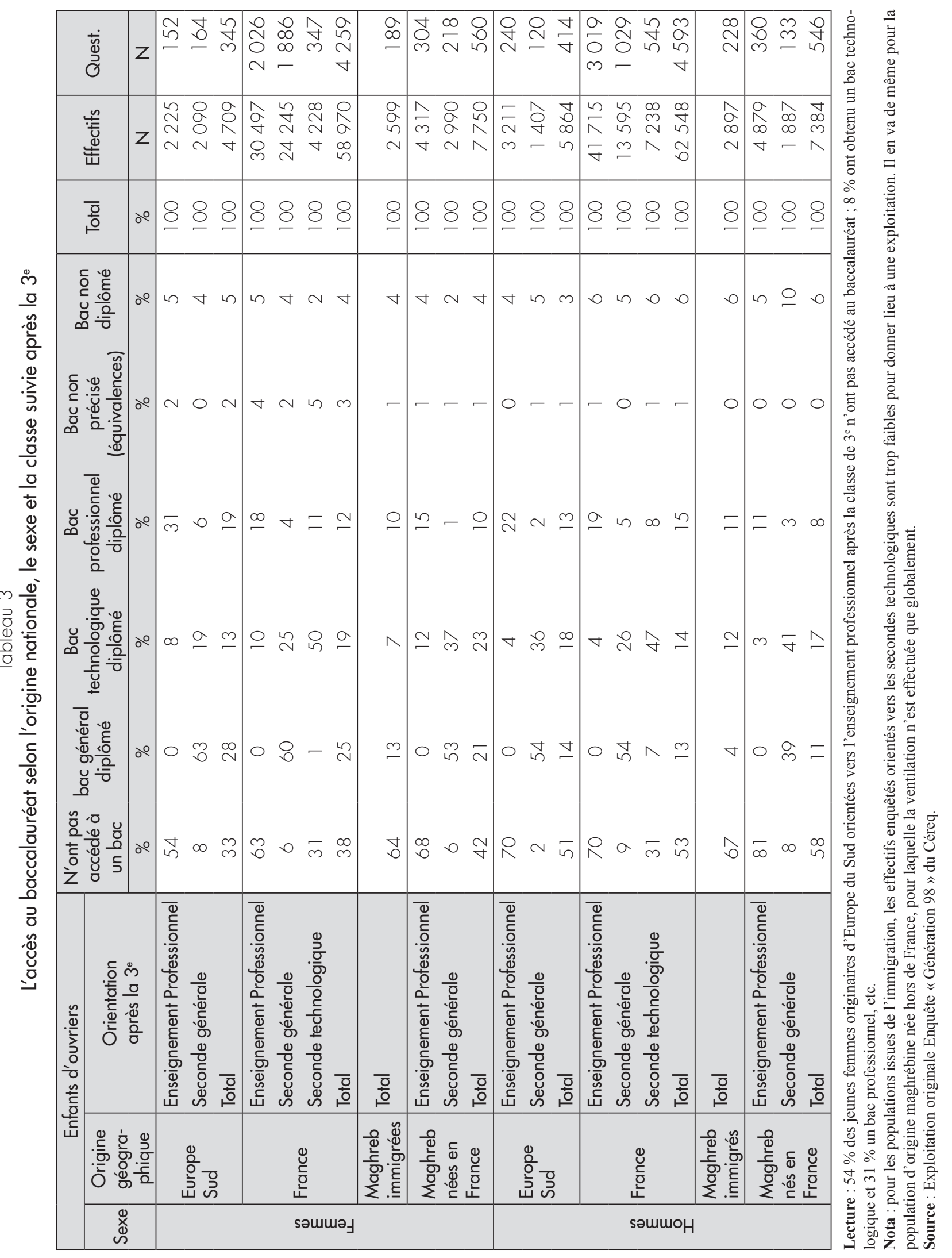


d'enseignement supérieur. $88 \%$ des femmes et $87 \%$ des hommes nés de parents immigrés nord africains entreprennent des études supérieures à l'issue de leur certification secondaire. En comparaison, les jeunes d'origine sud européenne ou française ne sont qu'environ $68 \%$ à tenter l'aventure du supérieur $(63 \%$ pour les jeunes descendants de natifs français). Avec le viatique du baccalauréat, pratiquement tous les jeunes d'origine maghrébine s'inscrivent dans l'enseignement supérieur, à l'université le plus souvent. L'écart avec les autres jeunes est considérable. Il confirme l'aspiration de ces bacheliers, maintes fois exprimée dans les enquêtes, à s'orienter vers des scolarités longues. Ce surinvestissement dans les études de ces «bacheliers de première génération» (Caille et Lemaire, 2009) peut manifester une volonté de se démarquer de la condition ouvrière. Cette dernière est d'autant plus souvent rejetée que nombre de pères maghrébins appartiennent aux franges les plus fragilisées de la classe ouvrière et que leurs enfants sont fréquemment témoins de leur mise à l'écart du monde du travail, soit par la mise ou chômage du père à la suite de restructurations industrielles, soit par une mise en invalidité consécutive à la pénibilité physique des emplois exercés (Frickey, Primon, 2009).

Cette «aversion» des jeunes nés de parents nord africains envers le monde ouvrier est manifeste lorsque l'on retrace le parcours des jeunes orientés vers les baccalauréats professionnels qui, théoriquement, destinent à la vie active. Les jeunes bacheliers professionnels d'origine française ou sud européenne, hommes comme femmes, sont une minorité à poursuivre des études, plus des trois quarts d'entre eux s'étant dirigés vers la vie active. À l'opposé, une majorité de bacheliers professionnels d'origine nord africaine ont fait le choix de la poursuite des études, qu'ils soient de sexe masculin ou féminin. ${ }^{11}$

Pour les baccalauréats généraux, la norme est à la poursuite des études; c'est précisément ce que font pratiquement tous les jeunes d'origine nord africaine,

${ }^{11}$ Les effectifs de l'enquête des enfants d'ouvriers maghrébins concernant les bacheliers professionnels sont faibles; ils n'indiquent donc qu'une tendance. Cependant, si l'on considère l'ensemble de la population d'origine maghrébine (toutes origines sociales confondues), qui garde un caractère populaire très affirmé et dont on sait que les résultats scolaires ne sont pas supérieurs à ceux des enfants d'ouvriers, on constate une permanence des résultats énoncés. tout comme les autres jeunes. Enfin, lorsqu'ils sont titulaires d'un baccalauréat technologique, ces mêmes jeunes issus de l'immigration maghrébine sont de nouveau plus nombreux que les autres à poursuivre des études supérieures.

\section{... MAIS PEINENT À FRANCHIR L'OBSTACLE DE LA PREMIÈRE ANNÉE}

Cependant, malgré ce surengagement dans les études supérieures, les jeunes d'origine maghrébine sont les principaux déçus de la démocratisation scolaire (Beaud, 2002). Beaucoup d'entre eux ne se tournent vers l'université qu'après avoir été refusés dans les filières de l'enseignement supérieur court, préparant au BTS ou au DUT (brevet de technicien supérieur et diplôme universitaire de technologie). L'université peut donc n'être qu'un second choix. Cependant, près de $60 \%$ des jeunes d'origine nord africaine ayant fréquenté un établissement d'enseignement supérieur se sont dirigés vers les formations universitaires, principalement littéraires ou juridiques (contre $45 \%$ et $48 \%$ des jeunes d'origine française ou sud européenne). En première année d'université, ces «nouveaux étudiants» (Erlich, 1998) sont livrés à eux-mêmes face à l'anonymat des amphithéâtres, aux cours magistraux, à la liberté d'organisation de leur travail... En d'autres termes, dans un monde universitaire où les règles et normes sont souvent implicites, où le travail personnel est valorisé, les étudiants conservant un rapport secondarisé aux études, ne répondent pas aux attentes de l'institution et ne parviennent pas à franchir le cap de la première année de DEUG (diplôme d'études universitaires générales). Parmi ceux-ci, les bacheliers technologiques ou professionnels sont particulièrement nombreux (Frickey, Primon, 2000).

Dans l'enquête Génération 98, près des deux tiers des jeunes d'origine maghrébine, (64\%), nés de parents ouvriers, ne sont pas parvenus à passer l'obstacle de la première année d'études à l'université, contre environ un tiers des jeunes nés de parents d'origine française (37\%) ou sud européenne (33\%). Parmi les titulaires 
d'un baccalauréat technologique ou professionnel les trois quarts des jeunes d'origine maghrébine sont stoppés à l'entrée des études. Pour les jeunes d'origine maghrébine nés de père ouvrier qui se sont dirigés vers les études supérieures courtes (BTS, DUT), la première année d'études reste également très sélective : $45 \%$ d'entre eux quittent les études sans obtenir le BTS ou le DUT (contre moins d'un quart des jeunes d'origine française et moins d'un tiers des jeunes nés de parents sud européens). Dans l'enquête de terrain que nous avons menée auprès des répondants à l'enquête "Génération 98 », les jeunes d'origine nord africaine évoquent assez peu cet "écrémage 》 en termes collectifs, de discrimination envers un groupe.

Tableau 4

Le parcours post bac selon le type de bac, le sexe et l'origine nationale des parents.

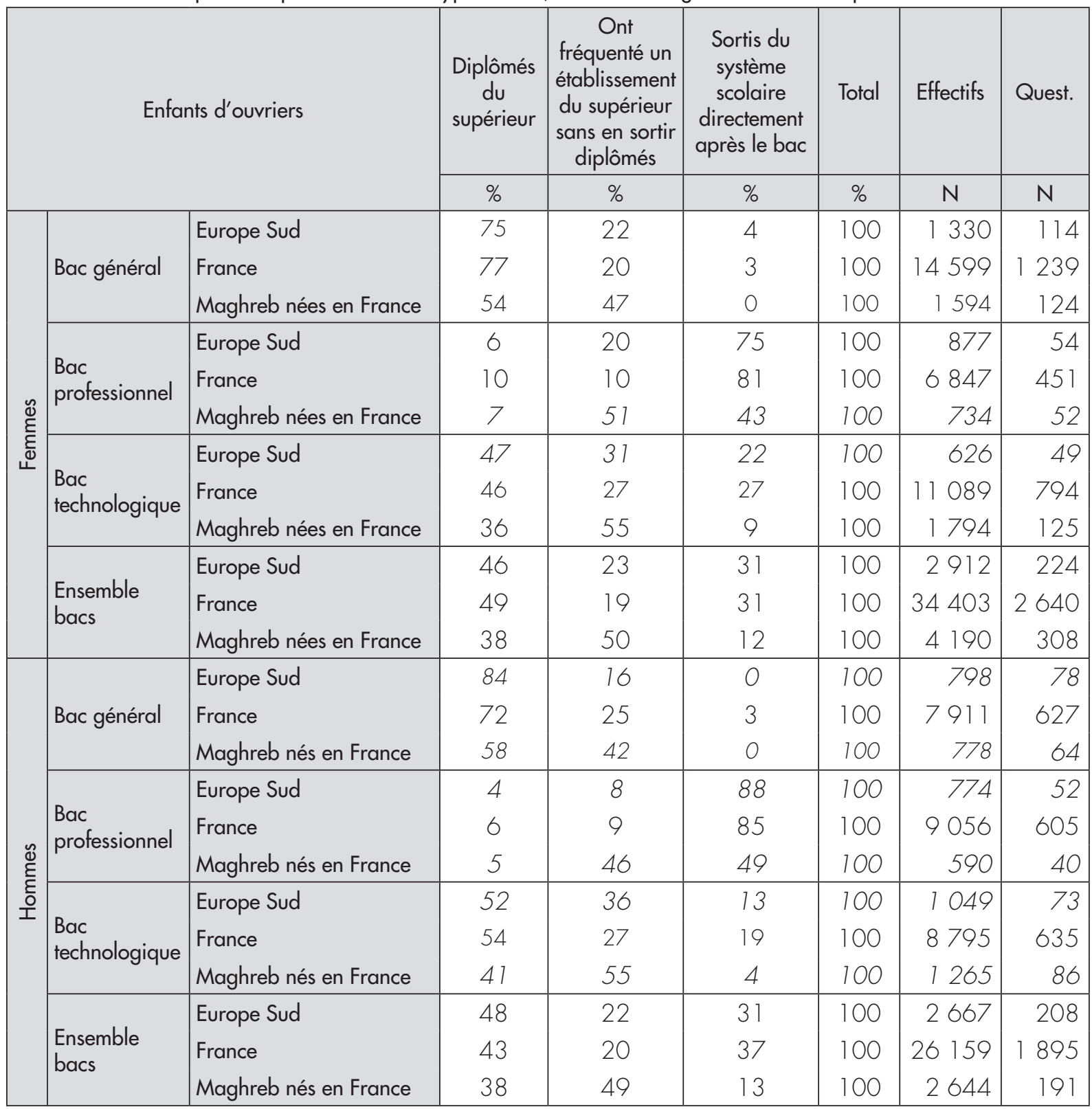

Lecture : parmi les jeunes femmes nées de parents originaires d'Europe du sud et titulaires d'un bac général, $75 \%$ possèdent un diplôme de l'enseignement supérieur, $22 \%$ sont sorties sans diplôme supérieur au bac, $4 \%$ sont entrées dans la vie active directement après le bac, etc.

Source : Exploitation originale Enquête « Génération 98 » du Cereq. 
Le registre le plus fréquemment utilisé rétrospectivement relève essentiellement du mode individuel ; l'échec est davantage vu comme un abandon, justifié à la fois par un manque d'intérêt envers des études trop théoriques et la nécessité ou l'opportunité d'un emploi, opportunité qu'il importait de saisir. Le discours dominant dans les médias sur le chômage des jeunes constitue également un argument en faveur de l'abandon d'études incertaines.

Avant de conclure sur cette mise en perspective des trajectoires scolaires des fils et filles d'ouvriers, d'origine immigrée ou descendants de natifs, nous tenterons de démêler les effets respectifs des facteurs pouvant intervenir aux différentes étapes du cursus scolaire.

\section{UNE ANALYSE LOGISTIQUE POUR NEUTRALISER LES EFFETS DE STRUCTURE}

Afin d'apprécier dans quelle mesure les différences observées dans les parcours au collège et au lycée puis à l'entrée de l'enseignement supérieur sont corrélées à l'origine nationale et au sexe, et de déterminer leurs effets respectifs, nous avons réalisé une régression logistique. Celle-ci neutralise les effets de structure associés aux populations immigrées ${ }^{12}$ et au sexe. Elle permet ainsi de déterminer dans quelle mesure ces deux variables sont en relation avec les parcours scolaires, et le sens de ces relations.

Outre l'origine immigrée et le sexe, nous avons introduit, dans le modèle, trois variables indiquant la position sociale des parents, une variable liée à la scolarité primaire (l'âge à l'entrée en sixième) ${ }^{13}$, ainsi qu'une

\footnotetext{
${ }^{12}$ Afin de simplifier le modèle, nous avons comparé la population issue de l'immigration des pays du Maghreb à la population dont les parents sont nés en France. D'autre part, certaines variables comme la taille de la fratrie ou le lieu de résidence plus précis auraient permis d'affiner le modèle, mais elles n'étaient pas disponibles dans l'enquête Génération 98.

${ }^{13}$ Nous avons effectué au préalable une régression logistique sur cette variable « âge à l'entrée en sixième ». Toutes choses égales par ailleurs, les jeunes filles ont 1.4 fois plus de chances d'entrer en sixième en avance ou à l'heure. Ce n'est pas le cas des jeunes originaires du Maghreb qui ont 2.1 fois moins de chances d'être à l'heure en sixième que les jeunes d'origine française. Une origine
}

variable de localisation géographique (province ou Île de France). Les variables testées caractérisent les parcours scolaires au sein du secondaire et à l'entrée du supérieur. La première se rapporte à la probabilité de sortir du système scolaire sans aucun diplôme, les deux suivantes sont relatives à l'orientation en classe de $3^{\mathrm{e}}$ : d'une part, avoir suivi une classe de $3^{\mathrm{e}}$ générale ou non ; d'autre part, avoir été redirigé (ou non) à ce niveau vers les dispositifs qui offrent une nouvelle chance aux élèves en grande difficulté scolaire. Trois autres variables concernent la scolarité à l'issue du collège : d'une part, avoir suivi (ou non) une classe de seconde générale et, d'autre part, avoir été orienté (ou s'orienter) vers une seconde technologique ; enfin, avoir été dirigé vers les classes de BEP-CAP (brevet d'études professionnelles et certificat d'aptitude professionnelle) de l'enseignement professionnel. La suivante s'intéresse au fait d'avoir ou non achevé le cursus entrepris. Quatre variables se rattachent au baccalauréat : être bachelier (ou non), être titulaire d'un bac général, technologique ou professionnel (ou non). Enfin, la dernière variable nous renseigne sur la probabilité de sortir de l'enseignement supérieur avec un diplôme ou non.

Les résultats de la régression confirment le rôle essentiel d'une bonne scolarité primaire, ou du moins d'une scolarité non entachée d'un ou plusieurs redoublements, sur la suite du parcours. Un parcours primaire "sans faute" n'annule pas les effets des autres variables. Etre de sexe féminin augmente les chances, toutes choses égales par ailleurs, de suivre un cursus général dans l'enseignement secondaire et de sortir diplômé de l'enseignement supérieur. Corrélativement, être de sexe féminin permet d'éviter l'orientation vers les filières professionnelles ou technologiques.

L'analyse logistique confirme qu'être né de parents nord africains n'a pas d'effet sur l'orientation en $3^{\mathrm{e}}$ : toutes choses égales par ailleurs, les descendants de maghrébins ont les mêmes chances d'accession en classe de $3^{\mathrm{e}}$ générale que les descendants de natifs. Même si l'on sait pertinemment que les années de

ouvrière, une mère inactive sont également des facteurs synonymes d'une réduction des chances d'entrer en $6^{\mathrm{e}}$ à l'âge «normal » (1.8 et 1.2). Avec les variables du modèle, toutes choses égales par ailleurs, il subsiste donc un effet de l'origine qui reste prégnant sur le déroulement du cursus primaire. 


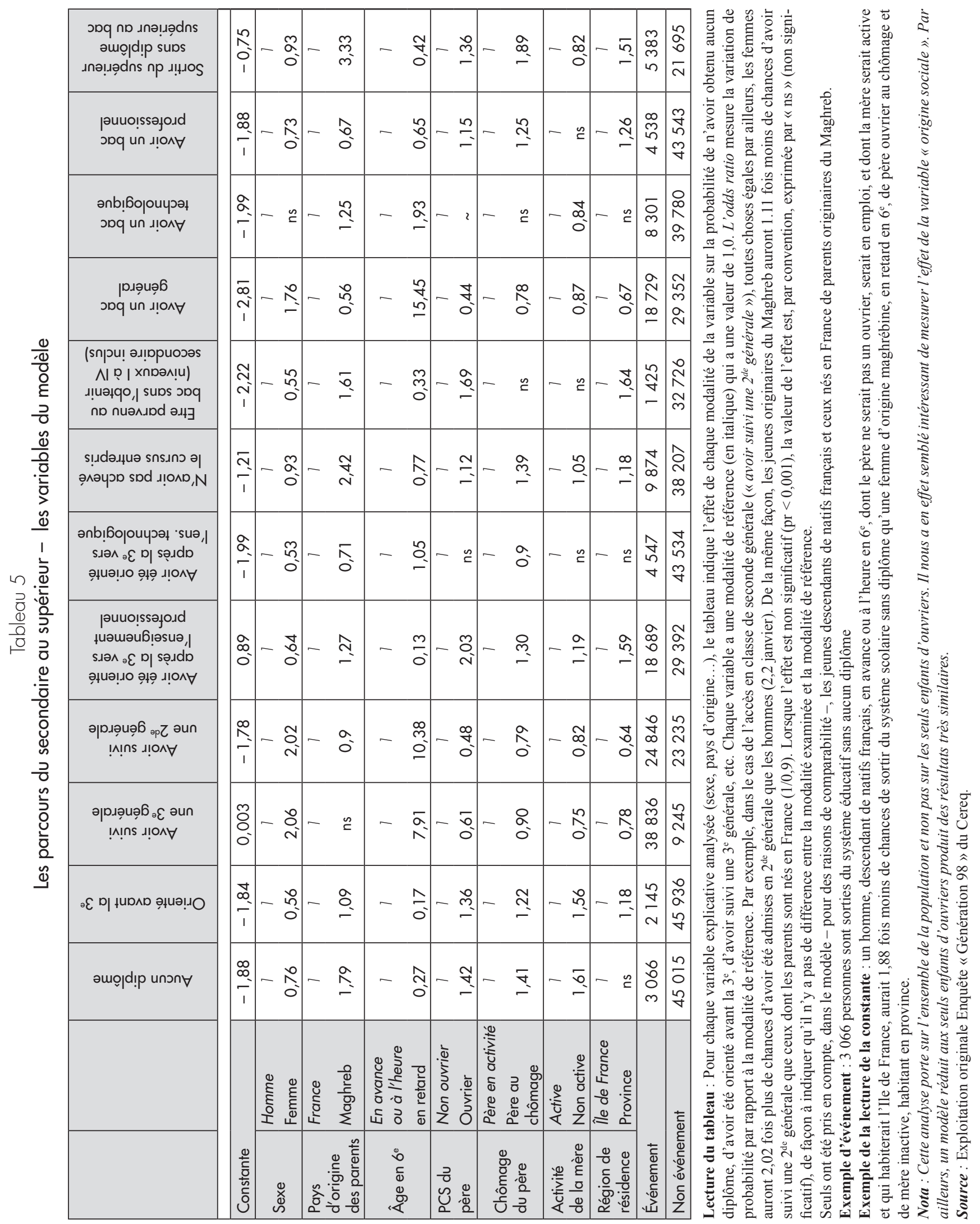


collège génèrent des inégalités de niveaux entre élèves ${ }^{14}$, ce n'est qu'après le « collège unique » que le processus d'orientation s'expose pleinement.

L'entrée en classe de seconde révèle des différences de rapports de chances entre descendants de natifs et descendants d'immigrés d'Afrique du Nord, au détriment de ces derniers. Mais ce qui caractérise le plus la variable « enfant d'immigré nord africain », c'est, d'une manière générale, la probabilité d'entreprendre un cycle d'études sans parvenir à l'achever. L'exemple le plus emblématique de cette propension à l'interruption des études reste la sortie du supérieur sans diplôme (probabilité 3,3 fois plus forte que pour les descendants de natifs). Cet effet subsiste, indépendamment de l'origine sociale du père, sachant qu'avoir un père ouvrier ou une mère inactive sont des variables qui jouent dans le même sens qu'une origine maghrébine sur les parcours scolaires.

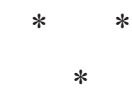

L'ensemble des données produites ci-dessus confirment une nouvelle fois que la catégorie «population immigrée ou issue de l'immigration » demeure trop hétérogène pour faire l'objet d'une analyse pertinente : aussi avons-nous distingué selon l'origine géographique de l'immigration et insisté sur les différences entre sexes. Ainsi, les cursus scolaires des jeunes originaires d'Europe du Sud et du Maghreb témoignent de cette hétérogénéité : les parcours des premiers s'apparentent davantage à ceux des jeunes d'origine française qu'à ceux de leurs homologues originaires, par leurs parents, d'Afrique du nord. À la lecture des résultats précédents, il apparaît également que l'appartenance à l'un ou l'autre sexe atténue ou renforce ces inégalités de parcours : d'une manière générale, dans les trois populations considérées, les jeunes femmes réussissent mieux à l'école que leurs homologues de sexe masculin ${ }^{15}$.

Bien entendu, en centrant l'analyse sur les seuls enfants d'ouvriers, et en excluant les jeunes nés

${ }^{14}$ L'analyse logistique met en évidence à la fois l'influence de facteurs scolaires et sociaux sur l'entrée en $3^{e}$ générale.

15 ... mais ces avancées sont toujours limitées par l'auto exclusion des filles des filières scientifiques, qu'elles soient d'origine immigrée ou non. au Maghreb, les différences observées tendent à se réduire, voire parfois à s'annuler. Il n'en demeure pas moins que d'autres, importantes, persistent, comme l'atteste par exemple le critère de l'âge à l'entrée en sixième. Les jeunes nés de parents ouvriers originaires des pays du Maghreb des deux sexes sont proportionnellement plus nombreux à connaître des difficultés scolaires dès le primaire, abordant ainsi le collège à un âge plus avancé. Cette variable est importante. On connaît en effet depuis longtemps l'importance d'une scolarité primaire sans accroc sur la suite du parcours.

Pour autant, à la sortie du collège, les données de l'enquête n'indiquent pas une orientation extrêmement marquée des jeunes originaires d'Afrique du nord vers les filières de l'enseignement professionnel. Ces résultats confirment les travaux récents de Caille pour lequel, à l'issue de la classe de $3^{\text {e }}$, le système scolaire n'oriente pas différemment et ne discrimine donc pas, à résultats scolaires équivalents, les jeunes d'origine immigrée (Caille, 2007). Cependant, les jeunes d'origine maghrébine, et notamment les hommes, interrogés dans le cadre d'interviews centrées sur la perception des discriminations, sont très nombreux à mettre en cause le système d'orientation. Ils expriment un profond sentiment d'injustice, sans toutefois aller jusqu'à faire état d'une discrimination exercée par l'institution ou par le corps enseignant envers eux. Ceci n'est guère surprenant tant il est difficile, dans le domaine des discriminations, de faire la part des choses, de démêler ce qui peut être attribué à l'appartenance de groupe, de ce qui relève des insuffisances scolaires individuelles. Il n'empêche, confrontés au processus d'orientation/sélection, ils subissent plutôt que choisissent la direction future de leurs études. Statistiquement, la plupart des études, et notamment celles menées par les services d'études du ministère de l'Éducation nationale, ne mettent en avant aucune différence d'orientation entre les jeunes d'origine immigrée et les jeunes descendants de français. Cependant, la persistance d'un sentiment d'injustice ressenti par les enfants de migrants nord africains interroge. Ne serait-ce pas en partie la conséquence du mode opératoire des enquêtes statistiques, privilégiant l'analyse « toutes choses égales par ailleurs » à la réalité concrète du terrain? Payet pose clairement la question de la portée d'un raisonnement qui établit que les jeunes d'origine immigrée réussissent aussi 
bien, voire mieux que les jeunes d'origine française, sachant que les établissements scolarisant les uns et les autres sont de plus en plus ségrégés socialement, ethniquement et spatialement. Dans ces conditions, c'est l'effet établissement qui prend le pas sur l'origine sociale (Payet, 2000). Cette ségrégation s'accentue à la fois par les stratégies d'évitement des établissements mal côtés, où les jeunes d'origine immigrée sont surreprésentés, et par le développement, au sein même de ces établissements, des classes de niveaux; celles-ci regroupant les élèves en « bonnes » et mauvaises » classes selon leurs résultats scolaires et/ou leur comportement vis-à-vis de l'école. Ces jeunes sont confrontés au quotidien à ce vécu scolaire là et non à une égalisation théorique des conditions sociales.

Les « jeux » ne sont pas encore entièrement faits à la sortie du collège. Cependant, les carrières scolaires des uns et des autres commencent à se dessiner assez nettement. En effet, une orientation en seconde générale équivaut à une forte probabilité d'obtenir un baccalauréat, et ce sont les jeunes femmes, d'origine française ou immigrée, qui s'engagent préférentiellement dans cette voie. À nouveau, les variations rela- tives aux pourcentages d'entrées en seconde générale, selon l'origine nationale des parents, se révèlent négligeables; mais alors que la plupart des jeunes entrés en seconde générale poursuivent leur route au sein des séries générales, une proportion importante de jeunes d'origine nord africaine entrés en seconde générale se retrouve orientée vers l'enseignement technologique, contribuant ainsi à leur surreprésentation dans ces filières du baccalauréat.

Enfin, un dernier point important caractérise les parcours scolaires des jeunes d'origine nord africaine : une forte propension à décrocher avant la fin du cycle d'études entrepris. Cette tendance est présente à tous les niveaux de sortie mais est particulièrement marquée à l'entrée de l'enseignement supérieur. Les enquêtes sur les projets d'avenir de ces jeunes ont toutes montré qu'à niveau scolaire égal, ils affichaient des ambitions plus élevées. De ce point de vue, leurs parcours révèlent qu'ils ont été au bout de leur démarche, en postulant, davantage que les autres jeunes, à un diplôme de l'enseignement supérieur, même lorsqu'ils n'étaient pas « objectivement » préparés à la condition d'étudiant. Leur désillusion n'en est que plus grande !

\section{Bibliographie}

Bastide H. (1984), Les enfants d'immigrés et l'enseignement français : enquête dans les établissements du ler et 2e degré, INED, Paris, PUF.

Baudelot C., Establet R. (1991), Allez les filles!, Paris, Seuil.

Beaud S. (2002), $80 \%$ au bac et après? Les enfants de la démocratisation scolaire, La Découverte.

Birnbaum Y., Kieffer A. (2005), « D’une génération à l'autre, les aspirations éducatives des familles immigrées : ambition et persévérance », Éducation et Formations $\mathrm{n}^{\circ} 72$, DEP, ministère de l'Éduca- tion nationale, de l'Enseignement supérieur et de la Recherche.

Caille J.-P., Lemaire S. (2009), «Les bacheliers de "première génération": des trajectoires scolaires et des parcours dans l'enseignement supérieur "bridés" par de moindres ambitions? », Données sociales.

Caille J.-P. (2007), « Perception du système éducatif et projets d'avenir des enfants d'immigrés ", Éducation et Formations $\mathrm{n}{ }^{\circ} 74$, DEP, ministère de l'Éducation nationale, de l'Enseignement supérieur et de la Recherche. 
Duru-Bellat M. (1990), L'École des filles. Quelles formations pour quels rôles sociaux?, Paris, L'Harmattan.

Erlich V. (1998), Les nouveaux étudiants, un groupe social en mutation, A. Colin.

Frickey A., Primon J.-L. (2000), «La socialisation des étudiants débutants Expériences universitaires familiales et sociales », Les Dossiers Éducation et Société, MEN, DPD, $\mathrm{n}^{\circ} 115$.

Frickey A., Primon J.-L. (2009), « Jeunes issus de l'immigration nord-africaine en fin de formation : une reproduction de la division des sexes? » L'Homme et la société, L'Harmattan.

Frickey A. (dir.) (2010), Projet ANR «Discriminations ressenties et inégalités sociales : des données statistiques aux récits biographiques ».

Marry C. (2001), «Filles et garçons à l'école» in Laufer J., Marry C., Maruani M. (dirs), Masculin Féminin: questions pour les sciences de l'homme, Paris, PUF.
Payet J.-P. (2000), «Violence à l'école et ethnicité : les raisons pratiques d'un amalgame», Ville-ÉcoleIntégration $\mathrm{n}^{\circ} 121$.

Passeron J.-C., Singly F. (1984), « Différences dans la différence : socialisation de classe et socialisation sexuelle », Revue Française de Science Politique, n ${ }^{\circ}$, vol. 4.

Passeron J.-C. (1991), Le raisonnement sociologique. L'espace non poppérien du raisonnement naturel, Paris, Nathan, «Essais et Recherches ».

Vallet L.-A., Caille J.-P. (1996), « Les élèves étrangers ou issus de l'immigration dans l'école et le collège français. Une étude d'ensemble », Les dossiers d'Éducation et Formations, 67, ministère de l'Éducation nationale, DEP.

Zeroulou Z. (1988) «La réussite scolaire des enfants d'immigrés. L'apport d'une recherche en termes de mobilisation ", Revue française de sociologie, XXIX-3.

Zirotti J.-P. (1980), La scolarisation des enfants de travailleurs immigrés. Taxinomies et situations scolaires, université de Nice, IDERIC.

\section{Résumé}

\section{Les inégalités de parcours scolaires des enfants d'origine maghrébine résultent-elles de discriminations? \\ Alain Frickey}

Cet article s'intéresse aux parcours scolaires des jeunes issus de l'immigration. II se focalise sur les différents points de bifurcation qui jalonnent la scolarité des jeunes. L'objectif est de savoir si les destinées scolaires des jeunes d'origine immigrée sont davantage structurées par leur sexe que par leur origine; si les inégalités à l'école se cumulent pour les hommes d'origine immigrée; et enfin si les inégalités constatées ou ressenties peuvent être assimilées à des discriminations. Pour répondre à ces questions, l'article s'appuie à la fois sur l'enquête "Génération 98 » du Céreq qui a interrogé, en 2001, un échantillon de 54000 jeunes de tous niveaux sortis de la formation initiale trois ans plus tôt, et sur des entretiens biographiques menés auprès de certains de ces jeunes.

Mots clés :

Cheminement scolaire, inégalité des chances, population d'origine étrangère, enquête génération 98, accès à l'enseignement supérieur

Journal of Economic Literature : I 21, J 15 